\title{
Plasma ghrelin levels in healthy elderly volunteers: the levels of acylated ghrelin in elderly females correlate positively with serum IGF-I levels and bowel movement frequency and negatively with systolic blood pressure
}

\author{
T Akamizu ${ }^{1}$, T Murayama ${ }^{4}$, S Teramukai ${ }^{3}$, K Miura $^{2}$, I Bando ${ }^{4}$, \\ T Irako' ${ }^{1}$ H Iwakura ${ }^{1}$, H Ariyasu ${ }^{1}$, H Hosoda ${ }^{1,8}$, H Tada $^{3}$, \\ A Matsuyama $^{3}$, S Kojima ${ }^{3}$, T Wada ${ }^{5}$, Y Wakatsuki ${ }^{5}$, \\ K Matsubayashi ${ }^{6}$, T Kawakita ${ }^{7}$, A Shimizu ${ }^{2}$, M Fukushima ${ }^{3}$, \\ $\mathbf{M ~ Y o k o d e ~}^{\mathbf{3}}$ and $\mathbf{K}$ Kangawa ${ }^{\mathbf{1 , 8}}$ \\ ${ }^{1}$ Ghrelin Research Project and ${ }^{2}$ Post-genome Project, Department of Experimental Therapeutics, Kyoto University Hospital, 54 Shogoin-kawaharacho, \\ Sakyo-ku, Kyoto 606-8507, Japan \\ ${ }^{3}$ Department of Clinical Innovative Medicine, and ${ }^{4}$ Department of Clinical Trial Design and Management, Kyoto University Hospital, Kyoto 606-8507, Japan \\ ${ }^{5}$ Translational Research Center, Kyoto University Hospital, and Department of Geriatric Medicine, Kyoto University School of Medicine, \\ Kyoto 606-8507, Japan \\ ${ }^{6}$ Center for Southeast Asian Studies, Kyoto University, Kyoto 606-8501, Japan \\ ${ }^{7}$ Kyoto Preventive Medical Centre, Kyoto 604-8491, Japan \\ ${ }^{8}$ Department of Biochemistry, National Cardiovascular Center Research Institute, Osaka 565-8565, Japan \\ (Requests for offprints should be addressed to T Akamizu; Email: akamizu@kuhp.kyoto-u.ac.jp)
}

\begin{abstract}
Aging is associated with a decrease in growth hormone $(\mathrm{GH})$ secretion, appetite and energy intake. As ghrelin stimulates both $\mathrm{GH}$ secretion and appetite, reductions in ghrelin levels may be involved in the reductions in GH secretion and appetite observed in the elderly. However, only preliminary studies have been performed on the role of ghrelin in elderly subjects. In this study, we sought to clarify the physiologic implications of the age-related alterations in ghrelin secretion by determining plasma ghrelin levels and other clinical parameters in healthy elderly subjects. Subjects were $\geq 65$ years old, corresponding to the SENIEUR protocol, had not had a resection of the upper gastrointestinal tract and had not been treated with hormones. One hundred and five
\end{abstract}

volunteers (49 men and 56 women) were admitted to this study $(73 \cdot 4 \pm 6 \cdot 3$ years old). Plasma levels of acylated ghrelin in elderly female subjects positively correlated with serum IGF-I levels and bowel movement frequency and negatively with systolic blood pressure. In elderly men, desacyl ghrelin levels correlated only weakly with bowel movement frequency. These findings suggest that the plasma levels of the acylated form of ghrelin may influence the age-related alterations in GH/IGF-I regulation, blood pressure and bowel motility. These observational associations warrant further experimental studies to clarify the physiologic significance of these effects.

Journal of Endocrinology (2006) 188, 333-344

\section{Introduction}

Aging is associated with progressive decreases in growth hormone $(\mathrm{GH})$ secretion, appetite and energy intake (Wurtman et al. 1988, Corpas et al. 1993, Morley 1997, Muller et al. 1999). This reduced GH secretion is termed 'somatopause' and may be a cause of age-related metabolic and physiologic changes, including reduced lean body mass and expansion of adipose mass. Altered blood lipid profiles also favor the development of vascular diseases that may increase overall mortality. The age-related reduction in energy intake has been termed 'the anorexia of aging' and predisposes to the development of undernutrition (Morley 1997). Common in older people, undernutrition has been implicated in the development and progression of chronic diseases commonly affecting the elderly, as well as in increasing mortality (Wurtman et al. 1988).

The mechanisms underlying the reduced GH secretion in aged animals and humans are complex (Muller et al. 1999, 
Chapman 2000). Age-related changes appear to involve the function of hypothalamic peptides specifically regulating GH secretion, and GH-releasing hormone (GHRH) and somatostatin (SS), appear to play a major role in this event. Experimental evidence indicates that within the rat hypothalamus, GHRH synthesis is impaired with increased age; relative hyperfunction of the SS-ergic system is also found in this animal. The physiologic causes of the anorexia of aging are largely unknown and probably multifactorial (Morley 1997). Possible mechanisms include a reduction in the central and/or peripheral feeding drives and increased activity of central and/or peripheral satiety signals (Martinez et al. 1993, Morley 1997, de Jong et al. 1999).

Ghrelin, a 28-amino-acid peptide, exhibits a variety of actions, including vasorelaxation (Nagaya et al. 2001, Shimizu et al. 2003) and stimulation of GH secretion (Takaya et al. 2000, Arvat et al. 2001, Hataya et al. 2001), appetite (Korbonits et al. 2004, van der Lely et al. 2004) and gastrointestinal motility (Masuda et al. 2000, Trudel et al. 2002, Fujino et al. 2003). A portion of ghrelin possesses a unique fatty acid modification, n-octanoylation, at Ser 3 (Kojima et al. 1999). Of the two circulating forms of ghrelin, acylated and unacylated (desacyl), the acylated form is thought to be essential for ghrelin biologic activity. Recently, however, desacyl ghrelin was reported to influence both cell proliferation and adipogenesis (Cassoni et al. 2001, Bedendi et al. 2003, Broglio et al. 2004, Thompson et al. 2004), prompting us to hypothesize that alterations in ghrelin may be involved in the reduction of $\mathrm{GH}$ secretion and appetite in elderly subjects. Preliminary studies using small numbers of elderly subjects demonstrated that the mean plasma concentrations of total ghrelin in normal weight geriatric subjects were lower than those present in younger, normal-weight subjects (Rigamonti et al. 2002, Sturm et al. 2003). In addition, $\mathrm{GH}$ response to ghrelin administration in elderly subjects is lower than that seen in young subjects (Broglio et al. 2003). While ghrelin mRNA levels in the stomach gradually decrease with increasing age in rats, serum levels of total ghrelin did not exhibit obvious age-related variation (Liu et al. 2002). In contrast, studies in rat indicated that both stomach ghrelin secretion and ghrelin-induced GH secretion increased in aged rats in comparison to younger rats (Englander et al. 2004). Total ghrelin secretion also increases with aging in monkeys (Angeloni et al. 2004). Although the disparity between humans and other animal models may be due to species differences, the number of human subjects was rather small. In addition, no adjustment of plasma ghrelin levels by other parameters was attempted, leaving the results of these human studies in question.

In this study, we determined the plasma concentrations of the two ghrelin forms, acylated and desacyl ghrelin, and their relationship to various anthropometric, hormonal and metabolic parameters in 105 elderly volunteers.
Using these measurements and appropriate analyses, we sought to clarify the age-related alteration in ghrelin secretion and the associated physiologic implications in elderly subjects.

\section{Materials and Methods}

\section{Subjects}

One hundred and thirty-seven (62 male and 75 female) elderly volunteers were registered for our study. Thirtytwo that did not satisfy the criteria for this study were excluded. Finally, 105 (49 male and 56 female) volunteers aged 65-94 years were subjected to analysis. All of the subjects were Japanese, and were recruited from the outpatient clinics of Kyoto University Hospital $(n=66$ (male, $n=36$; female, $n=30)$ ) and Kyoto Preventive Medical Center $(n=39$ (male, $n=13$; female, $n=26)$ ). The inclusion criteria were as follows: $1 . \geq 65$ years of age; 2 . correspondence with the SENIEUR protocol (Ligthart et al. 1984); 3. provision of written consent to participate in this study. Patients with either past history of upper gastrointestinal tract resection or present use of either hormones or steroids were excluded. The SENIEUR protocol provides strict admission criteria for human immunogerontologic studies. This protocol used clinical information (infection, inflammation, malignancy and other conditions, including acute myocardial infarction, treated cardiac insufficiency, hypertension of arteriosclerotic or diabetic origin, dementia, pregnancy, malnutrition, alcoholism and drug abuse), laboratory data (erythrocyte sedimentation rate, hemoglobin levels, mean corpuscular volume, leukocyte count with differentiation, immunoelectrophoresis, urinalysis and serum concentrations of urea, alkaline phosphatase, glucose, ASAT, ALAT and protein) and pharmacologic interference (prescribed medication for the treatment of the disorders defined above, anti-inflammatory drugs, hormones and analgesics) (Ligthart et al. 1984). This study included two exclusion criteria (no past resection of the upper gastrointestinal tract and no current treatment with hormones or steroids) to optimize endocrinologic and metabolic examination of stomach-derived hormones. The subjects who met all criteria were recognized as healthy subjects. Younger subjects, in whom plasma ghrelin levels were used for comparison with those in elderly subjects, were described previously (Akamizu et al. 2005). They were 16 male and 20 female Japanese volunteers 21-61 years of age. None of the subjects suffered from any known medical conditions or were currently taking medication. The period of the study was from March to September 2004. The study protocol was approved by the ethics committees on human research of Kyoto University Graduate School of Medicine and Kyoto Preventive Medical Center. Written, informed consent was obtained from all subjects prior to enrollment. 


\section{Laboratory analyses and biomedical factors}

Blood samples for hormone and glucose analyses were drawn from a forearm vein in the morning after overnight fast. Plasma samples were prepared as previously described (Kojima et al. 1999, Akamizu et al. 2005). Blood samples were immediately transferred to chilled polypropylene tubes containing EDTA-2Na $(1 \mathrm{mg} / \mathrm{ml})$ and aprotinin (Ohkura Pharmaceutical, Kyoto, Japan: 500 kallikrein inactivator $\mathrm{U} / \mathrm{ml}$ ), were centrifuged at $4{ }^{\circ} \mathrm{C}$. We added $1 \mathrm{~N} \mathrm{~mol} / 1 \mathrm{HCl}$ (10\% volume of plasma volume) to the separated plasma immediately. Plasma levels of acylated and unacylated ghrelin were measured with two commercially available ELISA kits, the Active Ghrelin ELISA and Desacyl-Ghrelin ELISA respectively, according to the manufacturer's protocol (Mitsubishi Kagaku Iatron, Tokyo, Japan) (Akamizu et al. 2005). The minimal detection limits for acylated and desacyl ghrelin in this assay system were 2.5 and $12.5 \mathrm{fmol} / \mathrm{ml}$ respectively. The intraand interassay coefficients of variation were $6.5 \%$ and $9.8 \%$ for acylated ghrelin and $3 \cdot 7 \%$ and $8 \cdot 1 \%$ for desacyl ghrelin respectively. Ghrelin measurements of samples from the older and young subjects were performed with the same kits, but not in the same assay. Plasma glucose was measured by the glucose oxidase method. Serum GH, insulin-like growth factor (IGF)-I and insulin concentrations were measured by immunoradiometric assay (IRMA), while serum leptin levels were measured by RIA (Mitsubishi Kagaku Bio-Clinical Laboratories, Tokyo, Japan). Insulin resistance was calculated according to the homeostasis model of assessment of insulin resistance (HOMA-IR), calculated as insulin $(\mu \mathrm{U} / \mathrm{ml}) \times$ blood glucose (mmol/l)/22.5 (Haffner et al. 1997).

The questionnaire presented to all subjects collected information about their sleeping time duration, bowel movements, smoking habits, alcohol consumption, use of medication, past medical history and physical activity. The question about bowel frequency was, 'How often do you usually defecate - once a day, more than once a day or once per 2 or 3 days?'

\section{Statistical analysis}

Data are expressed as the mean \pm S.D. We used Student's $t$-test to compare the means of the variables measured in both groups. The relationships between ghrelin concentrations and the variables studied were assessed by multiple regression analysis. The variables examined in the multiple regression models were site of recruitment, gender, age or age group (elderly and younger group), body-mass index (BMI), sleeping duration and blood levels of GH, IGF-I, insulin, glucose and leptin. The associations between ghrelin concentrations and blood pressure or bowel movement were assessed by multiple regression analysis after adjustment by the potential confounding factors according to gender. As the ghrelin distribution was slightly skewed, natural logarithms of ghrelin were used for the regression analysis. To identify the subsets of parameters that are statistically significantly related to each hormone level, we performed multiple regression analysis with a backward-elimination procedure after adjustment for the potential effect of site. All statistical analyses were performed by SAS, Version 8.02 (SAS Institute, Cary, NC, USA). $P$ values less than 0.05 were considered to be statistically significant.

\section{Results}

\section{Plasma ghrelin concentrations in elderly subjects}

We examined the anthropometric, hormonal and metabolic parameters of elderly volunteers (Table 1 ). The levels of acylated ghrelin in plasma were not significantly different between male and female subjects, while plasma levels of desacyl ghrelin in female subjects were significantly higher than those observed in male subjects (male, 53.3 \pm $41.5 \mathrm{fmol} / \mathrm{ml}$; female, $72 \cdot 0 \pm 46 \cdot 1 \mathrm{fmol} / \mathrm{ml} ; P=0.031)$. In comparison to our previous study of younger volunteers (mean age $=33.5 \pm 9 \cdot 0, n=36) \quad($ Akamizu et al. 2005), plasma levels of acylated ghrelin in elderly female subjects were significantly reduced from the levels in younger female subjects $(11.9 \pm 9.8$ vs $19.9 \pm 9.8 \mathrm{fmol} / \mathrm{ml} ; P=$ 0.004) (Fig. 1A and B). We did not observe any significant differences in the plasma levels of acylated ghrelin in men $(9 \cdot 3 \pm 11 \cdot 6$ vs $10 \cdot 9 \pm 6 \cdot 1 \mathrm{fmol} / \mathrm{ml})$ or desacyl ghrelin levels in both sexes (male, $53 \cdot 3 \pm 41.5$ vs $49 \cdot 1 \pm$ $23.5 \mathrm{fmol} / \mathrm{ml}$; female, $72 \cdot 0 \pm 46 \cdot 1$ vs $79 \cdot 8 \pm 53 \cdot 9 \mathrm{fmol} /$ $\mathrm{ml}$ ) between the elder and younger subjects. The ratios of acylated to desacyl ghrelin (A/D ratio) in elderly female subjects were significantly lower than those in younger female subjects $(16 \cdot 3 \pm 8 \cdot 2$ vs $26 \cdot 8 \pm 7 \cdot 8 ; P=0 \cdot 001)$. Men did not exhibit any significant differences between the age groups $(18 \cdot 0 \pm 11 \cdot 0$ vs $22 \cdot 6 \pm 8 \cdot 8 ; P=0 \cdot 101)$ (Fig. 1C). Age, BMI, insulin, leptin and HOMA-IR levels, however, also differed significantly between the sexes. In addition, the volunteers were recruited from two independent sites. Although nearly all of the parameters, including ghrelin levels, did not differ significantly between the sites, the $\mathrm{A} / \mathrm{D}$ ratios in both sexes and diastolic blood pressure (BP) values in men were significantly different (Table 1). To account for these differences, recruitment site, gender and age group were included as independent variables in the multiple regression analyses.

\section{Correlations of ghrelin concentrations with various parameters in elderly subjects}

In contrast to the results in Student's $t$-test, plasma levels of acylated ghrelin in women were not correlated with age group in the multiple regression analyses $(P=0.914)$ (Table 2 ), suggesting that those in elderly female subjects 


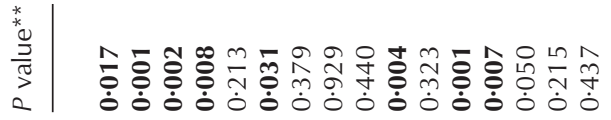

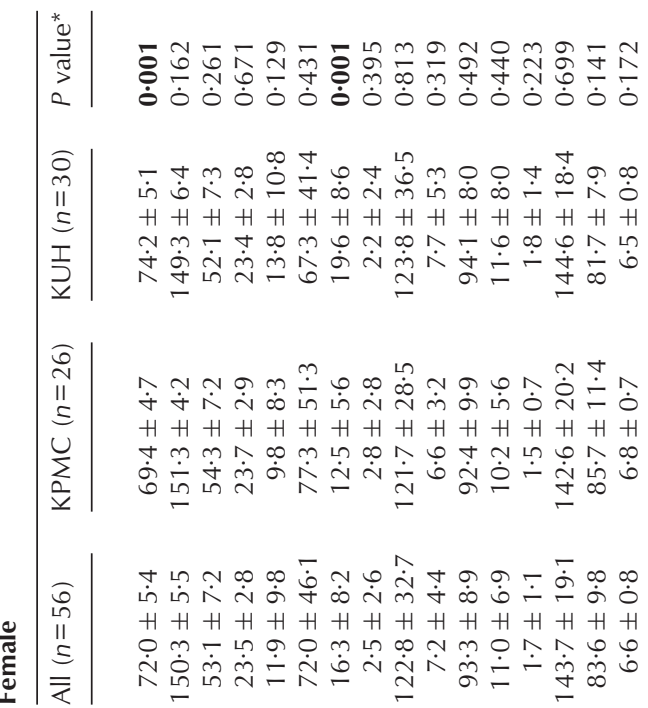

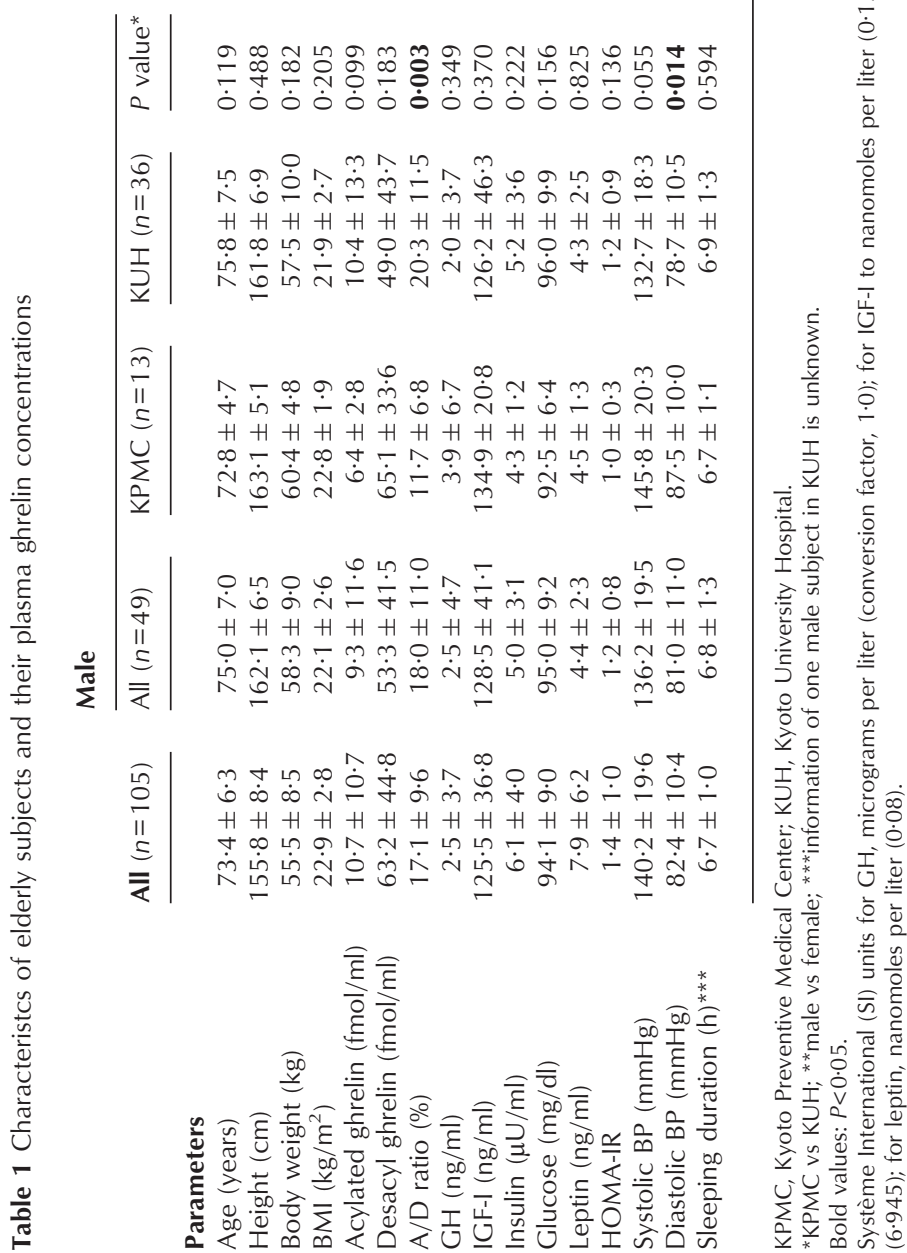



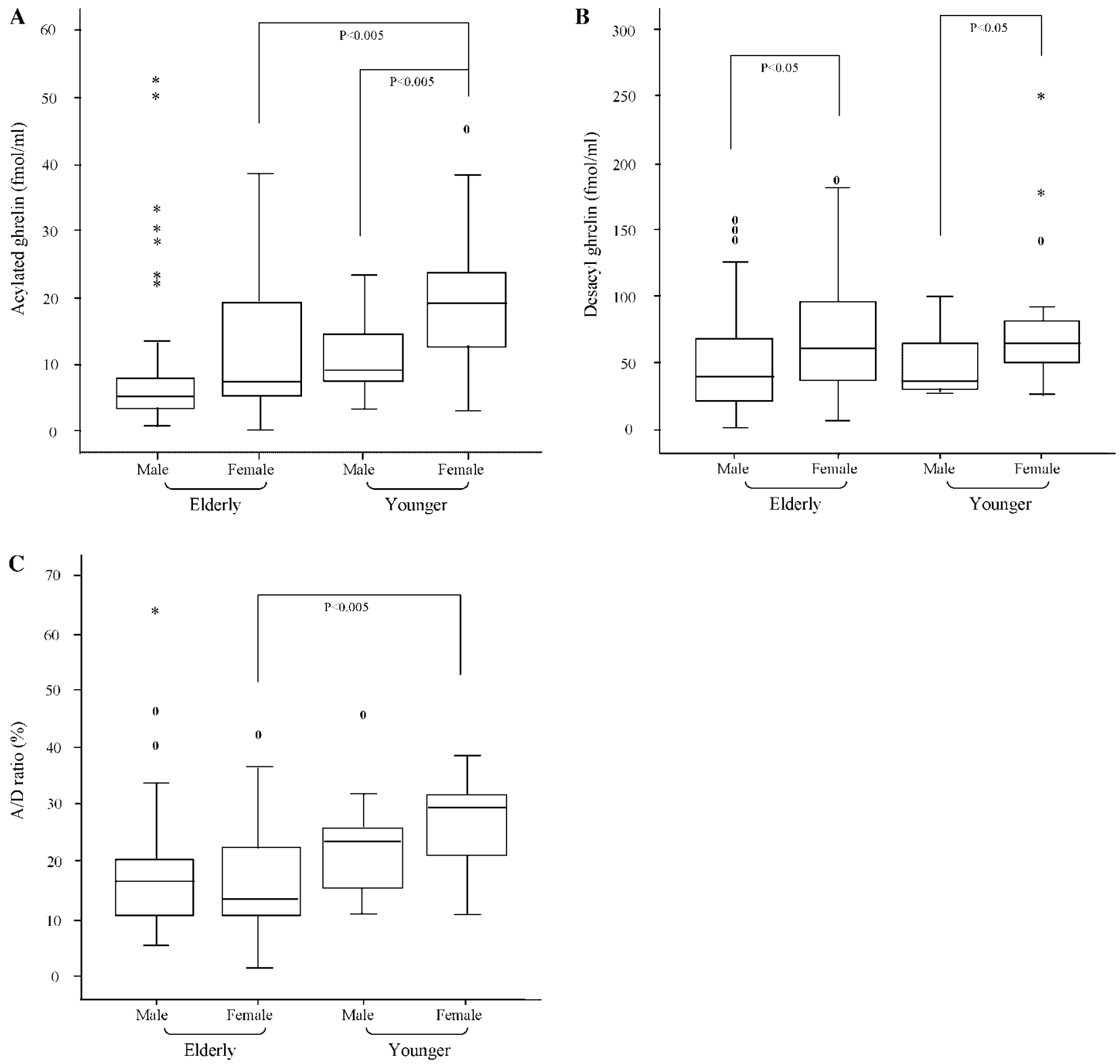

Figure 1 Comparison of plasma acylated ghrelin levels (A), desacyl ghrelin levels (B) and (C) A/D ratios between elderly and younger subjects. The values for subjects younger than 65 years are derived from our previous studies (Akamizu et al. 2005). Results are shown as box and whiskers plots. The upper hinge of the box represents the 75th and the lower the 25th percentile. The median is shown as a line across the box. The whiskers above and below the boxes represent the largest and smallest observed scores that are less than 1.5 box lengths from the box. Values farther away are potential outliers. If zero (0) appears, the value is between 1.5 and 3 interquartile ranges from the top or bottom edge of the box. If an asterisk $\left(^{*}\right)$ appears, the value is more extreme.

were not significantly different from those in younger female subjects. In addition, plasma levels of desacyl ghrelin in elderly subjects were not associated with gender $(P=0 \cdot 175)$. Although age and blood levels of $\mathrm{GH}$ and insulin were correlated with plasma levels of acylated and/or deacyl ghrelin in younger subjects, no parameter correlated with them in elderly subjects or elderly males. In elderly females, however, acylated ghrelin levels positively correlated with IGF-I $(P=0 \cdot 010)$. As this positive correlation between ghrelin and IGF-I levels was surprising, we examined the interaction between BMI and IGF-I by dividing female subjects into two groups based on the median value of BMI, 23·3. Acylated ghrelin levels correlated significantly with IGF-I levels in the group with lower $(<23 \cdot 3) \quad(P=0 \cdot 014)$, but not higher $(>23 \cdot 3)$ $(P=0 \cdot 090)$, BMI values (Fig. 2$)$. We did not observe any 

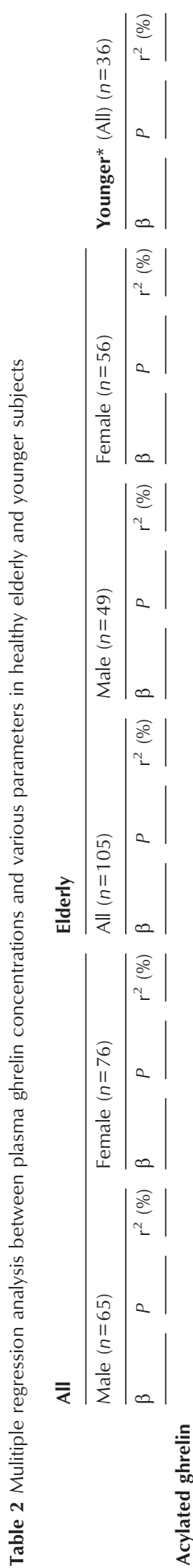

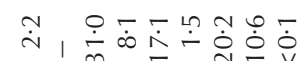

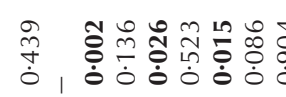

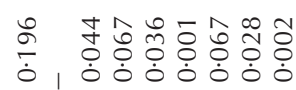

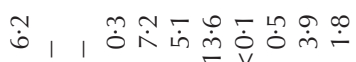

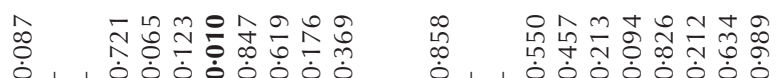

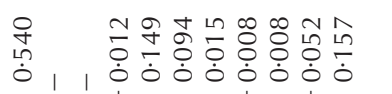

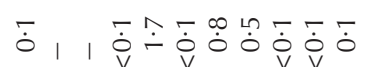

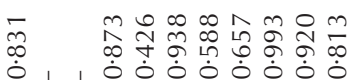

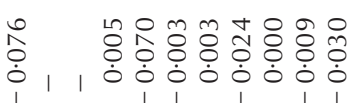

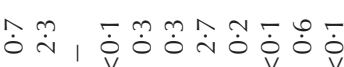

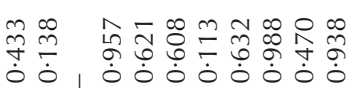

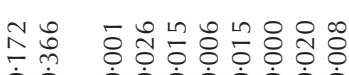

ó

|

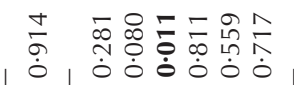

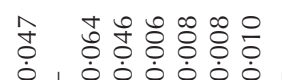

| |

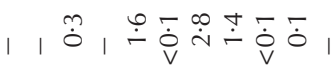

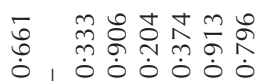

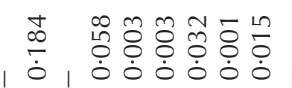

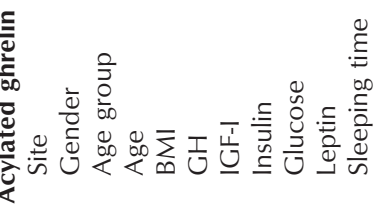

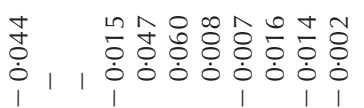

|

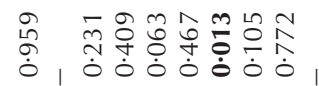

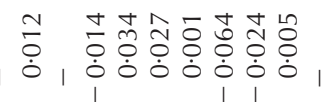

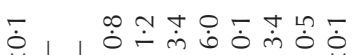

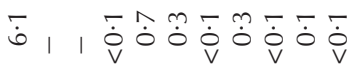

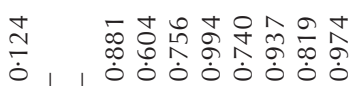

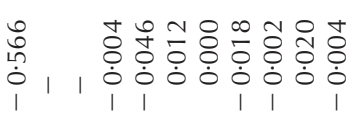

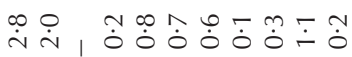

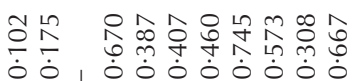

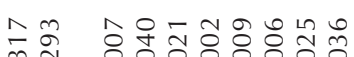

$\dot{0} \dot{0}, \dot{0} \dot{0} \dot{0} \dot{0} \dot{0} \dot{0} \dot{0} \dot{0}$

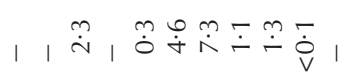

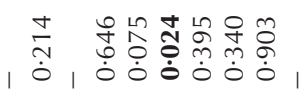

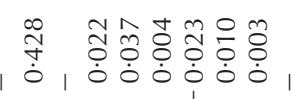

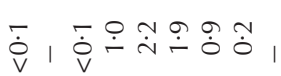

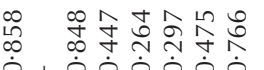

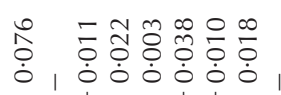

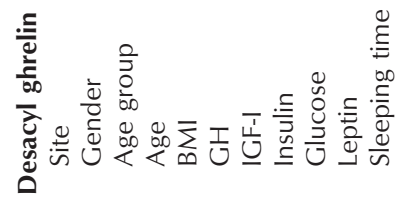




\section{A Female, BMI $<23.3(n=28)$}

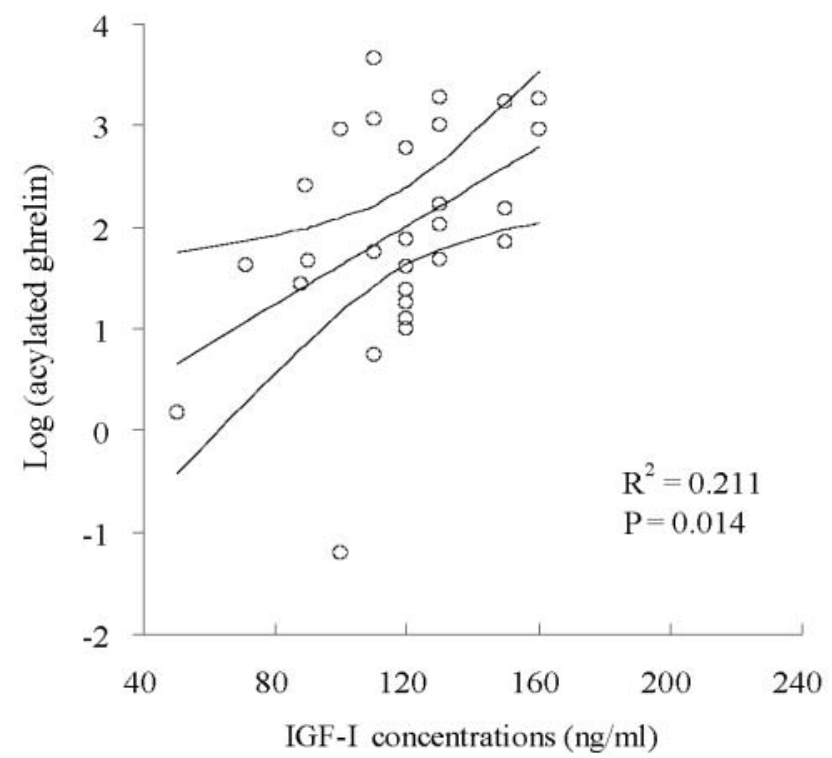

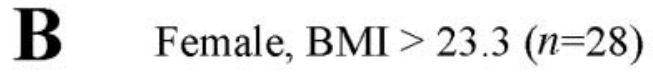

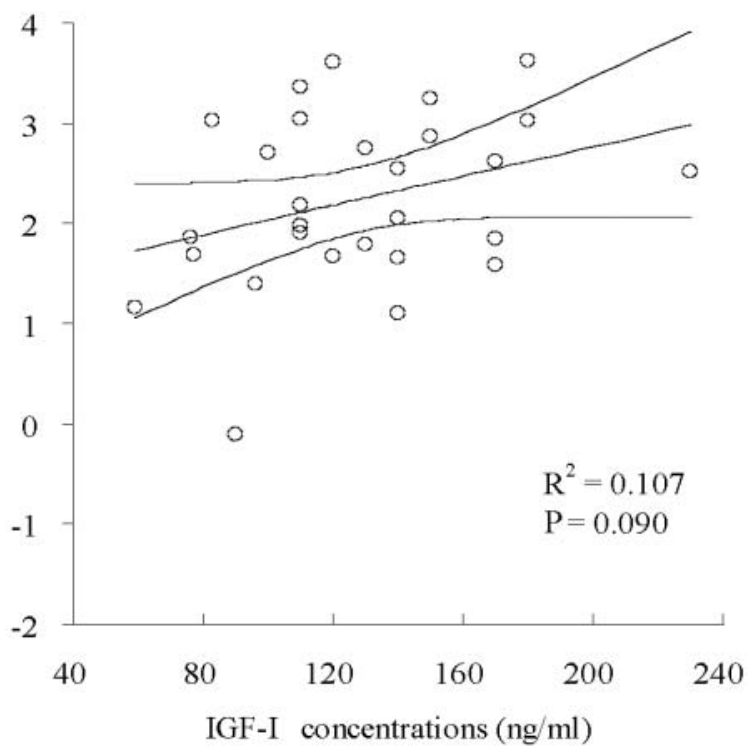

Figure 2 Linear regression analysis of the relationship between IGF-I and plasma acylated ghrelin levels in elderly female subjects of differing BMI levels. (A) Subjects with lower BMI $(<23 \cdot 3, n=28)$; (B) those with higher BMI $(>23 \cdot 3, n=28)$.

significant correlations between either acylated ghrelin and GH levels or GH and IGF-I levels in the group with a lower BMI. Although statistically not significant, plasma levels of acylated and desacyl ghrelin in elderly female subjects tended to be positively associated with BMI, while those in elderly men tended to be negatively associated (Table 2 and Fig. 3).

In the multivariate model, acylated ghrelin levels in women, but not in men, correlated with systolic BP levels, independently of site, age, BMI, sleeping duration,

\section{A Female, acylated ghrelin vs. BMI}

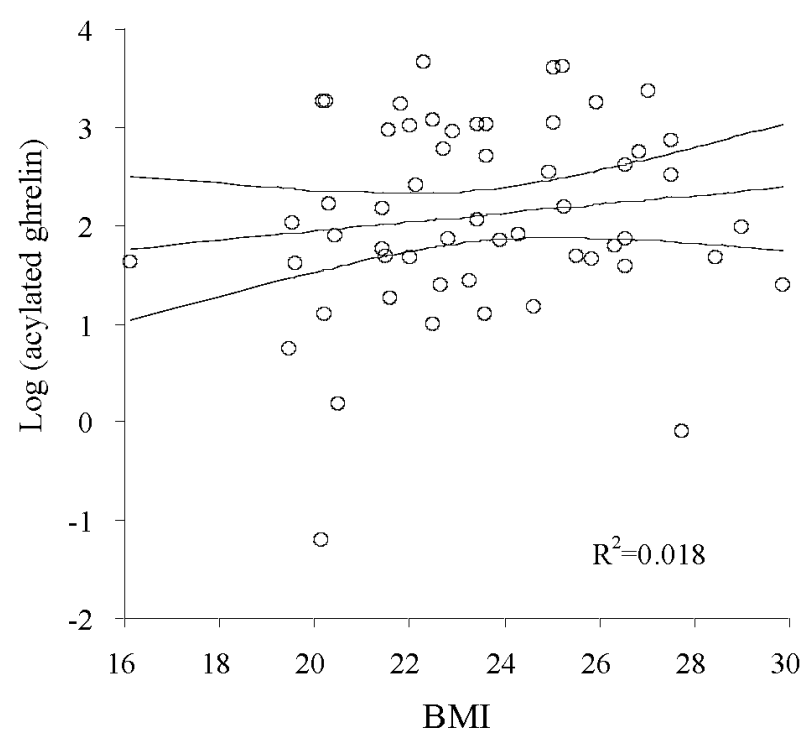

\section{B Female, desacyl ghrelin vs. BMI}

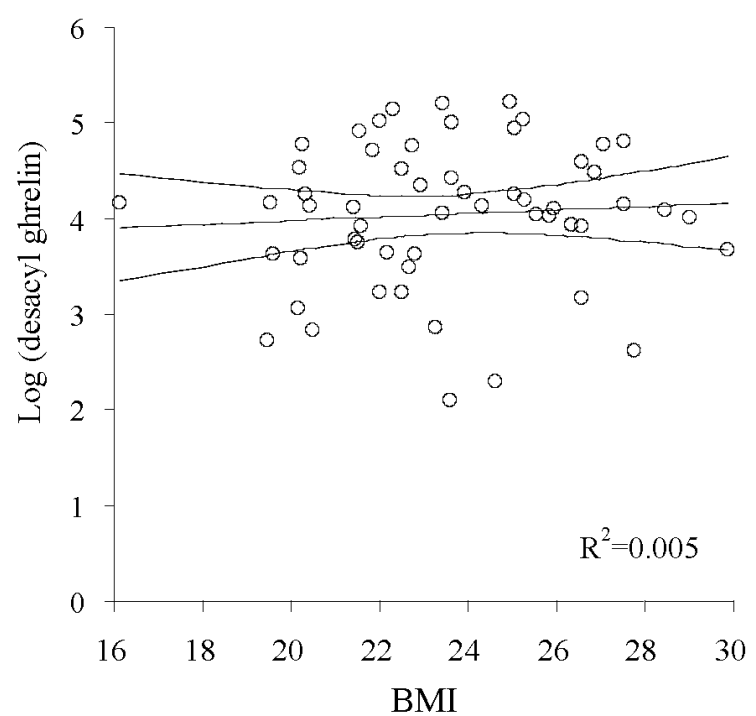

Figure 3 Linear regression analysis of the relationship between BMI and plasma levels of acylated (A) or desacyl (B) ghrelin in elderly female subjects. 
Table 3 Relationship between plasma ghrelin concentrations and blood pressure in healthy elderly subjects

\begin{tabular}{|c|c|c|c|c|c|c|}
\hline & \multicolumn{2}{|c|}{ Acylated ghrelin } & \multicolumn{2}{|c|}{ Desacyl ghrelin } & \multicolumn{2}{|l|}{$\mathrm{A} / \mathrm{D}$ ratio } \\
\hline & $\bar{\beta}$ & $P^{*}$ & $\beta$ & $P^{*}$ & $\bar{\beta}$ & $P^{*}$ \\
\hline \multicolumn{7}{|l|}{ Male } \\
\hline Systolic blood pressure & -0.003 & $0 \cdot 789$ & $0 \cdot 009$ & $0 \cdot 467$ & $-0 \cdot 012$ & $0 \cdot 050$ \\
\hline Diastolic blood pressure & $0 \cdot 012$ & $0 \cdot 616$ & $-0 \cdot 016$ & $0 \cdot 525$ & $0 \cdot 028$ & $0 \cdot 027$ \\
\hline \multicolumn{7}{|l|}{ Female } \\
\hline Systolic blood pressure & -0.022 & $0 \cdot 039$ & -0.016 & 0.074 & $-0 \cdot 006$ & $0 \cdot 291$ \\
\hline Diastolic blood pressure & -0.007 & $0 \cdot 719$ & -0.001 & 0.969 & -0.007 & $0 \cdot 565$ \\
\hline
\end{tabular}

*Adjusted by recruitment site, age, BMI, sleeping duration, blood pressure (mutually) and blood levels of GH, IGF-I, insulin, glucose and leptin.

Bold values: $P<0 \cdot 05$.

diastolic BP levels and blood levels of GH, IGF-I, insulin, glucose and leptin $(P=0 \cdot 039)$ (Table 3). Finally, acylated ghrelin levels and A/D ratio in women, but not in men, also correlated significantly with frequencies of bowel movement $(P=0 \cdot 014$ and $P=0 \cdot 008$ respectively) (Table 4). In men, desacyl ghrelin levels correlated with this parameter $(P=0 \cdot 037)$. There were no significant correlations between ghrelin levels and smoking habits in any subject groups. Gender difference was not an independent determinant of plasma ghrelin levels; $\beta$ and $P$ values for sex were $\beta=0.366$ and $P=0.138$ (acylated), and $\beta=0 \cdot 293$ and $P=0 \cdot 175$ (desacyl) respectively.

\section{Other hormone levels}

The correlations between other hormone levels and physiologic parameters in healthy elderly subjects are summarized in Table 5. Significantly, in both sexes, serum GH and IGF-I levels correlated negatively with BMI and age respectively, while serum leptin levels correlated positively with BMI. Plasma glucose levels positively correlated with both serum IGF-I levels and age. Serum IGF-I levels in females correlated positively with plasma concentrations of acylated ghrelin and negatively with serum GH levels, while serum leptin levels in men were significantly associated with age.

\section{Discussion}

Although two studies have demonstrated that mean plasma concentrations of total ghrelin in elderly, normalweight subjects were 36\% (Rigamonti et al. 2002) and $20 \%$ (Sturm et al. 2003) lower than those seen in younger, normal-weight subjects, these studies used a small number of subjects. In addition, only total ghrelin levels were examined, and no attempt was made to investigate gender differences. In this study, we demonstrated that the

Table 4 Relationship between plasma ghrelin concentrations and bowel movement in healthy elderly subjects

\begin{tabular}{|c|c|c|c|c|c|c|c|}
\hline & \multirow[b]{2}{*}{ Number } & \multicolumn{2}{|c|}{ Acylated ghrelin } & \multicolumn{2}{|c|}{ Desacyl ghrelin } & \multicolumn{2}{|c|}{$\mathrm{A} / \mathrm{D}$ ratiio } \\
\hline & & Mean & S.D. & Mean & S.D. & Mean & S.D. \\
\hline \multicolumn{8}{|l|}{ Bowel movement (male) } \\
\hline $1 /$ day & 29 & $9 \cdot 4$ & $13 \cdot 1$ & $52 \cdot 7$ & $41 \cdot 4$ & $17 \cdot 8$ & $10 \cdot 3$ \\
\hline$<1 /$ day & 5 & $4 \cdot 0$ & $3 \cdot 1$ & $27 \cdot 7$ & $34 \cdot 2$ & $27 \cdot 0$ & $21 \cdot 5$ \\
\hline $\begin{array}{l}P \text { value }{ }^{*} \\
\qquad(\geq 1 / \text { day vs }<1 / \text { day })\end{array}$ & & $0 \cdot 144$ & & $0 \cdot 037$ & & $0 \cdot 249$ & \\
\hline \multicolumn{8}{|c|}{ Bowel movement (female) } \\
\hline$\geq 2 /$ day & 11 & $15 \cdot 0$ & $9 \cdot 8$ & $91 \cdot 1$ & $43 \cdot 0$ & $15 \cdot 6$ & $4 \cdot 8$ \\
\hline $1 /$ day & 38 & $12 \cdot 3$ & $10 \cdot 2$ & $71 \cdot 3$ & $47 \cdot 8$ & $17 \cdot 3$ & $9 \cdot 0$ \\
\hline$<1 /$ day & 7 & $5 \cdot 5$ & $4 \cdot 4$ & $45 \cdot 2$ & $28 \cdot 6$ & $11 \cdot 8$ & $6 \cdot 1$ \\
\hline $\begin{array}{l}P \text { value }^{*} \\
\qquad(\geq 1 / \text { day vs }<1 / \text { day })\end{array}$ & & $0 \cdot 014$ & & $0 \cdot 188$ & & $0 \cdot 008$ & \\
\hline
\end{tabular}

*Adjusted by recruitment site, age, BMI, sleeping duration and blood levels of GH, IGF-1, insulin, glucose and leptin. Bold values: $P<0 \cdot 05$. 
Table 5 Mulitiple regression analysis between other hormone levels and various parameters in healthy elderly subjects*

\begin{tabular}{|c|c|c|c|c|c|c|}
\hline \multirow{3}{*}{$\begin{array}{l}\text { Hormone } \\
\text { GH }\end{array}$} & \multicolumn{3}{|l|}{ Male } & \multicolumn{3}{|l|}{ Female } \\
\hline & \multirow[t]{2}{*}{ Parameters } & \multirow[t]{2}{*}{$\beta$} & \multirow[t]{2}{*}{$P$} & \multirow[t]{2}{*}{ Parameters } & \multirow[t]{2}{*}{$\beta$} & \multirow[t]{2}{*}{$P$} \\
\hline & & & & & & \\
\hline & BMI & -0.971 & 0.001 & BMI & $-0 \cdot 411$ & $0 \cdot 001$ \\
\hline & Sleeping time & $1 \cdot 347$ & 0.007 & & & \\
\hline & Leptin & 0.610 & $0 \cdot 048$ & & & \\
\hline \multicolumn{7}{|l|}{ IGF-I } \\
\hline & Age & $-3 \cdot 262$ & $0 \cdot 001$ & Leptin & $3 \cdot 177$ & $0 \cdot 001$ \\
\hline & Glucose & $1 \cdot 540$ & 0.003 & Age & $-2 \cdot 317$ & $0 \cdot 002$ \\
\hline & Leptin & $4 \cdot 466$ & $0 \cdot 028$ & $\mathrm{BMI}$ & -5.987 & 0.004 \\
\hline & & & & Acylated ghrelin & 0.900 & $0 \cdot 012$ \\
\hline & & & & $\mathrm{GH}$ & $-3 \cdot 739$ & $0 \cdot 013$ \\
\hline \multicolumn{7}{|l|}{ Insulin } \\
\hline & BMI & 0.602 & $0 \cdot 001$ & Leptin & $0 \cdot 438$ & $0 \cdot 001$ \\
\hline \multicolumn{7}{|l|}{ Glucose } \\
\hline & IGF-I & 0.098 & 0.009 & IGF-I & $0 \cdot 128$ & 0.002 \\
\hline & Age & 0.528 & 0.012 & Age & 0.552 & $0 \cdot 040$ \\
\hline & $\mathrm{BMI}$ & $1 \cdot 006$ & $0 \cdot 048$ & & & \\
\hline \multicolumn{7}{|l|}{ Leptin } \\
\hline & BMI & $0 \cdot 513$ & $0 \cdot 001$ & $\mathrm{BMI}$ & $1 \cdot 410$ & $0 \cdot 001$ \\
\hline & Age & $0 \cdot 106$ & 0.017 & Insulin & 0.506 & 0.001 \\
\hline & & & & IGF-I & 0.044 & $0 \cdot 010$ \\
\hline
\end{tabular}

$\beta$ : regression coefficient.

* Multiple regression analysis with backward-elimination procedure was performed after adjustment for the effect of recruitment site; candidate independent parameters were age, BMI, sleeping duration and blood levels of GH, IGF-I, insulin, glucose and leptin.

plasma levels of acylated ghrelin in elderly male and female subjects were respectively $20 \%$ and $40 \%$ lower than those seen in younger subjects. In contrast, plasma concentrations of desacyl ghelin in elderly subjects of both sexes did not differ from those observed in younger subjects in both sexes. As a result, the A/D ratios in elderly female subjects were significantly lower than those in younger female subjects. In addition, plasma acylated ghrelin levels did not show significant gender difference, while plasma desacyl ghrelin levels in elderly female subjects were significantly higher than those in elderly male subjects, although gender difference was not an independent determinant for them. The reductions in acylated ghrelin levels observed in elderly female subjects may be partially related to a higher BMI than that seen in younger women $(23.5 \pm 2.9$ vs $20.3 \pm 1 \cdot 9 ; P<0.001)$, as plasma levels of acylated ghrelin in all females were not correlated with age group in the multiple regression analyses. Other modifying factors, especially menopause, should be considered as possibly affecting plasma acylated ghrelin levels in women. In support of this hypothesis, Kellokoski et al. (2005) recently reported that estrogen replacement therapy increases plasma levels of acylated ghrelin. Further studies will be necessary to delineate the mechanisms by which estrogen affects the production and/or secretion of acylated ghrelin.
ELISAs used for the measurement of plasma ghrelin levels in this study were two-site sandwich assays with two monoclonal antibodies. One monoclonal antibody recognizes the octanoyl-modified (Active Ghrelin kit) and the other the nonmodified N-terminal portion of ghrelin (Desacyl-Ghrelin kit) (Akamizu et al. 2005). The ratio of acylated to (acylated plus desacyl) ghrelin (A/(A+D) ratio) determined by ELISAs was lower than that of acylated to total ghrelin previously determined by RIA, which measures total ghrelin with an antiserum against the C-terminal region of ghrelin. This finding suggests that a fragmented form of ghrelin lacking the N-terminal region may naturally exist in human plasma or may be artificially produced during the RIA procedure. If so, then approximately $40-60 \%$ of the total ghrelin measured by RIA is probably fragmented. As a fragmented form of ghrelin is not measured in these two assays, its existence and physiologic implications should be considered and investigated in the future. A limitation of the study was that the measurements of ghrelin concentrations were not undertaken by inclusion of samples from both young and elderly subjects in the same assays. This increases the risk that interassay variation or drift may have reduced our ability to compare concentrations between the two age groups. To mitigate this risk, we used the same kind of assay kit. 
A negative correlation between BMI and plasma levels of total or acylated ghrelin was reported by many investigators, including us (Ariyasu et al. 2001, Tschop et al. 2001, Akamizu et al. 2005). On the contrary, plasma levels of both acylated and desacyl ghrelin in elderly female subjects tended to be associated positively with BMI, while those in elderly men tended to be negatively associated (Table 2 and Fig. 3). Particularly, the relationship between plasma acylated ghrelin levels and BMI should be noted, although statistically not significant $(\beta=0 \cdot 149, \quad P=0 \cdot 065)$. These findings suggest that the regulation of ghrelin secretion and/or production in elderly female subjects is altered in comparison to that seen in younger subjects. This altered regulation might be related to the anorexia and undernutrition associated with aging. For example, plasma ghrelin levels may not rise sufficiently when elderly subjects lose weight, resulting in poor appetite and a state of negative energy balance.

In women, acylated ghrelin concentrations correlated positively with IGF-I independently of recruitment site, age, BMI, sleeping duration or blood levels of GH, IGF-I, insulin, glucose and leptin. While a negative correlation between ghrelin and IGF-I levels was reported in children and adolescents (Bellone et al. 2002, Whatmore et al. 2003), such a correlation has not been observed in adult subjects (Dall et al. 2002, Malik et al. 2004). Recently, Poykkyo et al. (2005) reported a negative correlation between plasma ghrelin and IGF-I in adult subjects with obesity, insulin resistance and type 2 diabetes. The association was particularly strong in both men and subjects in the higher BMI tertiles (maximum: $29 \cdot 2$ or less). In women, the correlation disappeared in the lowest BMI tertile (minimum: $26 \cdot 5$ or more). In agreement with this report, we did not observe a significant correlation between plasma ghrelin and IGF-I levels in the higher BMI population $(>23 \cdot 3)$. Acylated ghrelin levels, however, correlated positively with IGF-I levels with lower BMI values $(<23 \cdot 3)$. The positive correlation of ghrelin and IGF-I observed in elderly subjects implicates the dysregulation of ghrelin secretion and/or production during aging, suggesting that the negative feedback regulation of IGF-I may be lost. The IGF-I levels observed in the lower BMI group, $117 \cdot 4 \pm 25 \cdot 1 \mathrm{ng} / \mathrm{ml}$, may be too low to inhibit ghrelin secretion. In this group, the positive correlation suggests that ghrelin regulates IGF-I production by affecting GH secretion. Although we could not identify significant correlations between either acylated ghrelin and GH levels or GH and IGF-I levels, such associations between plasma ghrelin levels and serum GH levels have been observed in previous studies (Yoshimoto et al. 2002, Akamizu et al. 2005). Thus, the regulation of ghrelin/GH/IGF-I axis in elderly women with low IGF-I levels may be different from that seen in the younger subjects with normal IGF-I levels.

Acylated ghrelin levels in elderly women correlated negatively with systolic BP. The inverse relationship between total ghrelin levels and BP has previously been reported in pregnant women (Makino et al. 2002) and patients with hypertension (Poykko et al. 2003). Ghrelin, which exerts vasorelaxant or vasodilatory effects in vitro (Okumura et al. 2002, Shimizu et al. 2003), decreases BP (Nagaya et al. 2001). Our study also demonstrated the novel correlation of ghrelin levels with frequency of bowel movement in elderly subjects. It should be noted that the smaller number of men than women might have resulted in the borderline correlation between male acylated ghrelin level and the frequency of bowel movement. Ghrelin administration in humans stimulates peristalsis (Takaya et al. 2000, Nagaya et al. 2001, Akamizu et al. 2004) and enhances gastric and intestinal motilities in rats (Masuda et al. 2000, Trudel et al. 2002, Fujino et al. 2003). These findings suggest that ghrelin might play a role in the regulation of bowel motility.

In this study, we confirmed that both blood IGF-I and glucose levels were significantly correlated with age (Davidson 1979, Corpas et al. 1993, Muller et al. 1999). Serum leptin levels, adjusted for various parameters including BMI, exhibited a significant positive association with age in men (Table 5), but a nonsignificant negative association with age in women (data not shown). This finding corresponds to a report by Baumgartner et al. (1999) suggesting that the differences among men and the changes with age in serum leptin levels are associated with differing circulating levels of testosterone. Although plasma glucose levels correlate positively with serum IGF-I levels, few investigators, as far as we know, have reported this positive correlation. As several regulatory factors affect both blood glucose and IGF-I levels, further investigations will be necessary to clarify the mechanisms underlying this correlation. Finally, we confirmed previously observed correlations between BMI and serum GH or leptin levels in elderly subjects (Baumgartner et al. 1999, Iranmanesh et al. 1991, Chapman 2004).

In summary, we measured plasma levels of acylated and desacyl ghrelin in healthy elderly subjects. The levels of acylated ghrelin in women correlated positively with IGF-I levels, suggesting that the negative feedback mechanism does not function properly in nonobese elderly subjects. These results suggest, however, that ghrelin may regulate IGF-I levels through control of GH. Acylated ghrelin concentrations in women correlated with both systolic BP and the frequency of bowel movements. These findings strongly suggest that, in elderly women, acylated ghrelin may play a role in the regulation of the GH/IGF-I axis, BP and bowel movements. The obvious next step is to explore and confirm these physiologic effects of ghrelin experimentally. In addition, analysis of 24-h acylated and desacyl ghrelin secretion is extremely important to determine the physiologic control of ghrelin secretion during the lifespan. Finally, understanding the relationship between plasma ghrelin levels and these clinical parameters in the elderly may provide therapeutic opportunities to target ghrelin in disorders related to aging. 


\section{Acknowledgements}

We thank Drs Hidenori Arai, Hisanori Horiuchi, Makoto Tanaka, Yutaka Nagano and Hiroshi Dekigai, Department of Geriatric Medicine, Kyoto University Hospital, for recruiting elderly subjects; Ms Hiratani, Ishimoto, and Takehisa for excellent technical assistance; and Ms Keiko Amemiya and Mizuho Hirata for excellent secretarial assistance. The authors declare that there is no conflict of interest that would prejudice the impartiality of this scientific work.

\section{Funding}

This study was supported by funds from the Ministry of Education, Science, Culture, Sports and Technology of Japan, and the Ministry of Health, Labor, and Welfare of Japan, and Research Grants by Chiyoda Foundation, Foundation for Growth Science, and Yamanouchi Foundation for Research on Metabolic Disorders.

\section{References}

Akamizu T, Takaya K, Irako T, Hosoda H, Teramukai S, Matsuyama A, Tada H, Miura K, Shimizu A, Fukushima M et al. 2004 Pharmacokinetics, safety, and endocrine and appetite effects of ghrelin administration in young healthy subjects. European Journal of Endocrinology 150 447-455.

Akamizu T, Shinomiya T, Irako T, Fukunaga M, Nakai Y, Nakai Y \& Kangawa K 2005 Separate measurement of plasma levels of acylated and desacyl ghrelin in healthy subjects using a new direct ELISA assay. Journal of Clinical Endocrinology and Metabolism 90 6-9.

Angeloni SV, Glynn N, Ambrosini G, Garant MJ, Higley JD, Suomi S \& Hansen BC 2004 Characterization of the rhesus monkey ghrelin gene and factors influencing ghrelin gene expression and fasting plasma levels. Endocrinology 145 2197-2205.

Ariyasu H, Takaya K, Tagami T, Ogawa Y, Hosoda K, Akamizu T, Suda M, Koh T, Natsui K, Toyooka S et al. 2001 Stomach is a major source of circulating ghrelin, and feeding state determines plasma ghrelin-like immunoreactivity levels in humans. Journal of Clinical Endocrinology and Metabolism 86 4753-4758.

Arvat E, Maccario M, Di Vito L, Broglio F, Benso A, Gottero C, Papotti M, Muccioli G, Dieguez C, Casanueva FF et al. 2001 Endocrine activities of ghrelin, a natural growth hormone secretagogue (GHS), in humans: comparison and interactions with hexarelin, a nonnatural peptidyl GHS, and GH-releasing hormone. Journal of Clinical Endocrinology and Metabolism 86 1169-1174.

Baumgartner RN, Waters DL, Morley JE, Patrick P, Montoya GD \& Garry PJ 1999 Age-related changes in sex hormones affect the sex difference in serum leptin independently of changes in body fat. Metabolism 48 378-384.

Bedendi I, Alloatti G, Marcantoni A, Malan D, Catapano F, Ghe C, Deghenghi R, Ghigo E \& Muccioli G 2003 Cardiac effects of ghrelin and its endogenous derivatives des-octanoyl ghrelin and des-Gln14-ghrelin. European Journal of Pharmacology 476 87-95.

Bellone S, Rapa A, Vivenza D, Castellino N, Petri A, Bellone J, Me E, Broglio F, Prodam F, Ghigo E et al. 2002 Circulating ghrelin levels as function of gender, pubertal status and adiposity in childhood. Journal of Endocrinological Investigation 25 RC13-RC15.

Broglio F, Benso A, Castiglioni C, Gottero C, Prodam F, Destefanis S, Gauna C, van der Lely AJ, Deghenghi R, Bo M et al. 2003 The endocrine response to ghrelin as a function of gender in humans in young and elderly subjects. Journal of Clinical Endocrinology and Metabolism 88 1537-1542.

Broglio F, Gottero C, Prodam F, Gauna C, Muccioli G, Papotti M, Abribat T, Van Der Lely AJ \& Ghigo E 2004 Non-acylated ghrelin counteracts the metabolic but not the neuroendocrine response to acylated ghrelin in humans. Journal of Clinical Endocrinology and Metabolism 89 3062-3065.

Cassoni P, Papotti M, Ghe C, Catapano F, Sapino A, Graziani A, Deghenghi R, Reissmann T, Ghigo E \& Muccioli G 2001 Identification, characterization, and biological activity of specific receptors for natural (ghrelin) and synthetic growth hormone secretagogues and analogs in human breast carcinomas and cell lines. Journal of Clinical Endocrinology and Metabolism 86 1738-1745.

Chapman IM 2000 Hypothalamic growth hormone-IGF-1 axis. In Endocrinology of Aging, pp 23-44. Eds JE Morley \& L van den Berg. Totowa, NJ, USA: Humana Press.

Chapman IM 2004 Endocrinology of anorexia of ageing. Best Practice and Research. Clinical Endocrinology and Metabolism 18 437-452.

Corpas E, Harman SM \& Blackman MR 1993 Human growth hormone and human aging. Endocrinology Review 14 20-39.

Dall R, Kanaley J, Hansen TK, Moller N, Christiansen JS, Hosoda H, Kangawa K \& Jorgensen JO 2002 Plasma ghrelin levels during exercise in healthy subjects and in growth hormone-deficient patients. European Journal of Endocrinology 147 65-70.

Davidson MB 1979 The effect of aging on carbohydrate metabolism: a review of the English literature and a practical approach to the diagnosis of diabetes mellitus in the elderly. Metabolism 28 688-705.

de Jong N, Mulder I, de Graaf C \& van Staveren WA 1999 Impaired sensory functioning in elders: the relation with its potential determinants and nutritional intake. Journals of Gerontology. Series A, Biological Sciences and Medical Sciences 54 B324-331.

Englander EW, Gomez GA \& Greeley GH Jr 2004 Alterations in stomach ghrelin production and in ghrelin-induced growth hormone secretion in the aged rat. Mechanisms of Ageing and Development 125 871-875.

Fujino K, Inui A, Asakawa A, Kihara N, Fujimura M \& Fujimiya M 2003 Ghrelin induces fasted motor activity of the gastrointestinal tract in conscious fed rats. Journal of Physiology 550 227-240.

Haffner SM, Miettinen H \& Stern MP 1997 The homeostasis model in the San Antonio Heart Study. Diabetes Care 20 1087-1092.

Hataya Y, Akamizu T, Takaya K, Kanamoto N, Ariyasu H, Saijo M, Moriyama K, Shimatsu A, Kojima M, Kangawa K et al. 2001 A low dose of ghrelin stimulates growth hormone $(\mathrm{GH})$ release synergistically with GH-releasing hormone in humans. Journal of Clinical Endocrinology and Metabolism 864552.

Iranmanesh A, Lizarralde G \& Veldhuis JD 1991 Age and relative adiposity are specific negative determinants of the frequency and amplitude of growth hormone (GH) secretory bursts and the half-life of endogenous GH in healthy men. Journal of Clinical Endocrinology and Metabolism 73 1081-1088.

Kellokoski E, Poykko SM, Karjalainen AH, Ukkola O, Heikkinen J, Kesaniemi YA \& Horkko S 2005 Estrogen replacement therapy increases plasma ghrelin levels. Journal of Clinical Endocrinology and Metabolism 90 2954-2963.

Kojima M, Hosoda H, Date Y, Nakazato M, Matsuo H \& Kangawa K 1999 Ghrelin is a growth-hormone-releasing acylated peptide from stomach. Nature 402 656-660.

Korbonits M, Goldstone AP, Gueorguiev M \& Grossman AB 2004 Ghrelin - a hormone with multiple functions. Front Neuroendocrinology 25 27-68.

Ligthart GJ, Corberand JX, Fournier C, Galanaud P, Hijmans W, Kennes B, Muller-Hermelink HK \& Steinmann GG 1984 Admission criteria for immunogerontological studies in man: the SENIEUR protocol. Mechanisms of Ageing and Development 28 47-55. 
Liu YL, Yakar S, Otero-Corchon V, Low MJ \& Liu JL 2002 Ghrelin gene expression is age-dependent and influenced by gender and the level of circulating IGF-I. Molecular and Cellular Endocrinology 189 97-103.

Makino Y, Hosoda H, Shibata K, Makino I, Kojima M, Kangawa K \& Kawarabayashi T 2002 Alteration of plasma ghrelin levels associated with the blood pressure in pregnancy. Hypertension $39781-784$.

Malik IA, English PJ, Ghatei MA, Bloom SR, MacFarlane IA \& Wilding JP 2004 The relationship of ghrelin to biochemical and anthropometric markers of adult growth hormone deficiency. Clinical Endocrinology $(\mathrm{Oxf}) 60$ 137-141.

Martinez M, Hernanz A, Gomez-Cerezo J, Pena JM, Vazquez JJ \& Arnalich F 1993 Alterations in plasma and cerebrospinal fluid levels of neuropeptides in idiopathic senile anorexia. Regulatory Peptides 49 109-117.

Masuda Y, Tanaka T, Inomata N, Ohnuma N, Tanaka S, Itoh Z, Hosoda H, Kojima M \& Kangawa K 2000 Ghrelin stimulates gastric acid secretion and motility in rats. Biochemical and Biophysical Research Communications 276 905-908.

Morley JE 1997 Anorexia of aging: physiologic and pathologic. American Journal of Clinical Nutrition 66 760-773.

Muller EE, Locatelli V \& Cocchi D 1999 Neuroendocrine control of growth hormone secretion. Physiology Review 79 511-607.

Nagaya N, Kojima M, Uematsu M, Yamagishi M, Hosoda H, Oya H, Hayashi Y \& Kangawa K 2001 Hemodynamic and hormonal effects of human ghrelin in healthy volunteers. American Journal of Physiology. Regulatory, Integrative and Comparative Physiology 280 R1483-R1487.

Okumura H, Nagaya N, Enomoto M, Nakagawa E, Oya H \& Kangawa K 2002 Vasodilatory effect of ghrelin, an endogenous peptide from the stomach. Journal of Cardiovascular Pharmacology 39 779-783.

Poykko SM, Kellokoski E, Horkko S, Kauma H, Kesaniemi YA \& Ukkola O 2003 Low plasma ghrelin is associated with insulin resistance, hypertension, and the prevalence of type 2 diabetes. Diabetes 52 2546-2553.

Poykko SM, Ukkola O, Kauma H, Kellokoski E, Horkko S \& Kesaniemi YA 2005 The negative association between plasma ghrelin and IGF-I is modified by obesity, insulin resistance and type 2 diabetes. Diabetologia 48 309-316.

Rigamonti AE, Pincelli AI, Corra B, Viarengo R, Bonomo SM, Galimberti D, Scacchi M, Scarpini E, Cavagnini F \& Muller EE 2002 Plasma ghrelin concentrations in elderly subjects: comparison with anorexic and obese patients. Journal of Endocrinology 175 R1-5.

Shimizu Y, Nagaya N, Teranishi Y, Imazu M, Yamamoto H, Shokawa T, Kangawa K, Kohno N \& Yoshizumi M 2003 Ghrelin improves endothelial dysfunction through growth hormone-independent mechanisms in rats. Biochemical and Biophysical Research Communications 310 830-835.

Sturm K, MacIntosh CG, Parker BA, Wishart J, Horowitz M \& Chapman IM 2003 Appetite, food intake, and plasma concentrations of cholecystokinin, ghrelin, and other gastrointestinal hormones in undernourished older women and well-nourished young and older women. Journal of Clinical Endocrinology and Metabolism 88 3747-3755.

Takaya K, Ariyasu H, Kanamoto N, Iwakura H, Yoshimoto A, Harada M, Mori K, Komatsu Y, Usui T, Shimatsu A et al. 2000 Ghrelin strongly stimulates growth hormone release in humans. Journal of Clinical Endocrinology and Metabolism 85 4908-4911.

Thompson NM, Gill DA, Davies R, Loveridge N, Houston PA, Robinson IC \& Wells T 2004 Ghrelin and des-octanoyl ghrelin promote adipogenesis directly in vivo by a mechanism independent of the type 1a growth hormone secretagogue receptor. Endocrinology 145 234-242.

Trudel L, Tomasetto C, Rio MC, Bouin M, Plourde V, Eberling P \& Poitras P 2002 Ghrelin/motilin-related peptide is a potent prokinetic to reverse gastric postoperative ileus in rat. American Journal of Physiology. Gastrointestinal and Liver Physiology 282 G948-G952.

Tschop M, Weyer C, Tataranni PA, Devanarayan V, Ravussin E \& Heiman ML 2001 Circulating ghrelin levels are decreased in human obesity. Diabetes 50 707-709.

van der Lely AJ, Tschop M, Heiman ML \& Ghigo E 2004 Biological, physiological, pathophysiological, and pharmacological aspects of ghrelin. Endocrinology Reviews 25 426-457.

Whatmore AJ, Hall CM, Jones J, Westwood M \& Clayton PE 2003 Ghrelin concentrations in healthy children and adolescents. Clinical Endocrinology (Oxf) 59 649-654.

Wurtman JJ, Lieberman H, Tsay R, Nader T \& Chew B 1988 Calorie and nutrient intakes of elderly and young subjects measured under identical conditions. Journal of Gerontology 43 B174-B180.

Yoshimoto A, Mori K, Sugawara A, Mukoyama M, Yahata K, Suganami T, Takaya K, Hosoda H, Kojima M, Kangawa K et al. 2002 Plasma ghrelin and desacyl ghrelin concentrations in renal failure. Journal of the American Society of Nephrology 13 2748-2752.

Received 21 July 2005

Accepted 27 October 2005

Made available online as an Accepted Preprint

18 November 2005 